University of Nebraska - Lincoln

DigitalCommons@University of Nebraska - Lincoln

Faculty Publications, Department of Physics and Astronomy

2013

\title{
Thermal electron attachment to F2
}

Nicholas S. Shuman

Thomas M. Miller

Albert A. Viggiano

Ilya I. Fabrikant

Follow this and additional works at: https://digitalcommons.unl.edu/physicsfacpub

This Article is brought to you for free and open access by the Research Papers in Physics and Astronomy at DigitalCommons@University of Nebraska - Lincoln. It has been accepted for inclusion in Faculty Publications, Department of Physics and Astronomy by an authorized administrator of DigitalCommons@University of Nebraska Lincoln. 


\title{
Thermal electron attachment to $\mathbf{F}_{2}$
}

\author{
Nicholas S. Shuman, ${ }^{1}$ Thomas M. Miller, ${ }^{1}$ Albert A. Viggiano, ${ }^{1, *}$ and Ilya I. Fabrikant ${ }^{2}$ \\ ${ }^{1}$ Space Vehicles Directorate, Air Force Research Laboratory, Kirtland Air Force Base, New Mexico 87117, USA \\ ${ }^{2}$ Department of Physics and Astronomy, University of Nebraska, Lincoln, Nebraska 68588-0299, USA
}

(Received 28 October 2013; published 11 December 2013)

\begin{abstract}
Rate constants have been measured from 300 to $700 \mathrm{~K}$ for thermal electron attachment to $\mathrm{F}_{2}$ using two flowing afterglow-Langmuir probe apparatuses. Dissociative attachment yielding $\mathrm{F}^{-}$is observed with a rate constant of $5.0 \pm 1.3 \times 10^{-9} \mathrm{~cm}^{3} \mathrm{~s}^{-1}$ at $300 \mathrm{~K}$, rising to $9.6 \pm 2.4 \times 10^{-9} \mathrm{~cm}^{3} \mathrm{~s}^{-1}$ at $700 \mathrm{~K}$, well below the previously accepted values of McCorkle et al. [D. L. McCorkle, L. G. Christophorou, A. A. Christodoulides, and L. Pichiarella, J. Chem. Phys. 85, 1966 (1986)]. The absolute concentration of $F_{2}$ reaching the afterglow is verified by measuring the near-collisional rate constant $\left(4.5 \pm 1.5 \times 10^{-10} \mathrm{~cm}^{3} \mathrm{~s}^{-1}\right)$ for $\mathrm{Ar}^{+}+\mathrm{F}_{2} \rightarrow \mathrm{ArF}^{+}+\mathrm{F}$. Prior attempts to apply $R$-matrix calculations to the $\mathrm{F}_{2}+e^{-}$system have failed to explain previously reported thermal and nonthermal attachment rate constants along with high-resolution, low-energy attachment cross sections. The present results are reproduced exceptionally well by $R$-matrix calculations employing previously calculated resonance widths without adjustment.
\end{abstract}

DOI: 10.1103/PhysRevA.88.062708

PACS number(s): $34.80 . \mathrm{Lx}, 34.80 . \mathrm{Ht}, 82.30 . \mathrm{Fi}$

\section{INTRODUCTION}

$\mathrm{F}_{2}$ is the simplest molecule that can undergo dissociative electron attachment (DEA) at thermal energies. The early reported existence $[1,2]$ of an $s$ wave $(l=0)$ type peak in low-energy DEA to $\mathrm{F}_{2}$, as evidenced by a cross-sectional energy dependence near threshold of $E^{-1 / 2}$, presented a challenge to theory, as the first temporary negative-ion state of $F_{2}$ has $\Sigma_{u}$ symmetry, and therefore should lead to $p$ wave $(l=1)$ type attachment with a cross section proportional to $E^{1 / 2}$ near threshold [3]. A similar difficulty in DEA to $\mathrm{Cl}_{2}$ was resolved [4] by accurate calculation of the potential energy curve for the $\Sigma_{u}$ state of $\mathrm{Cl}_{2}{ }^{-}$with subsequent calculation of DEA cross sections using the semiempirical $R$-matrix theory. It was shown that the crossing between the ground-state potential curve of $\mathrm{Cl}_{2}$ and the anion curve occurs to the left of the equilibrium internuclear separation for $\mathrm{Cl}_{2}$ (i.e., at smaller internuclear distance), and therefore the Franck-Condon factor for the transition from the neutral to the anion state drops quickly with the electron energy. This causes the $p$-wave peak to occur very close to zero electron energy, at about $50 \mathrm{meV}$, which appeared as an $s$-wave-type zero-energy peak in early experiments with insufficient energy resolution. Subsequent high-resolution experiments [5,6] agreed very well with the calculations, as did high-temperature thermal experiments [7].

An observation [8] of a low-energy peak in DEA to $F_{2}$ at an energy of $31 \mathrm{meV}$ suggested that perhaps earlier experiments also suffered from insufficient resolution or other artifacts, and that the same theoretical description successful for $\mathrm{Cl}_{2}$ would work for the attachment to $F_{2}$. However, all attempts to reproduce the low-energy peak in the DEA cross section have either failed to do so, or generated a very unrealistic anion potential energy curve [9]. A previous semiempirical semilocal calculation (i.e., nonlocal for the imaginary portion of the complex potential and local for the real portion) gave a peak position at $70 \mathrm{meV}$ [10]. Previous fully nonlocal

\footnotetext{
*Corresponding author: rvborgmailbox@kirtland.af.mil
}

calculations $[11,12]$ gave the peak position at energies between 170 and $200 \mathrm{meV}$. These values seem reasonable in comparison to the $\mathrm{Cl}_{2}$ peak at $50 \mathrm{meV}$ as the $\mathrm{F}_{2}$ molecule is lighter than $\mathrm{Cl}_{2}$ and therefore the energy dependence of the Franck-Condon factor is weaker, but are well above the experimental result of Braun et al. [8]. A recent local calculation [13] produces a very high (about $10^{-14} \mathrm{~cm}^{2}$ ) DEA cross section at zero energy. However, it has been well known [10,14] since the 1978 DEA calculations of Hall [15] that the local theory is inconsistent with the correct threshold behavior. We conclude that despite a 35-year history of theoretical attempts, differences with experimental data for this simple system remain large.

Whether the discrepancy is due to shortcomings in experiment, theory, or both is not immediately clear. Calculated cross sections differ substantially from each other [11-13]. Experimental thermal rate constants vary by an order of magnitude [16,17], and beam experiments have shown, each seemingly conclusively but obviously contradictory, either $s$-wave [1,2] or $p$-wave [8] behavior. Here, we aim to reconcile a portion of these disparate literature results, moving closer to solving the question of DEA to $\mathrm{F}_{2}$.

Thermal attachment rate constants are measured using flowing afterglow-Langmuir probe apparatuses over a temperature range of $300-700 \mathrm{~K}$. The results differ significantly from literature values, and are shown to be in excellent agreement with $R$-matrix calculations using previously reported calculations by Hazi et al. [11] of the resonance width and cross sections without adjustment.

\section{EXPERIMENTAL METHODS}

The measurements have been made using two flowing afterglow-Langmuir probe (FALP) apparatuses located at the Air Force Research Laboratory, with temperature ranges of 300-600 K (FALP) and 300-1200 K [high-temperature (HT)-FALP]. Other than differences in materials used to overcome issues for the higher temperature measurements, the instruments are similar to each other and have each been described in detail elsewhere [18-20]. Briefly, a weakly ionized plasma was created by discharging a 10-20 standard 
liter $\min ^{-1}$ flow of helium (99.999\%, Matheson) inside a microwave cavity. Downstream a few percent $(0.2-1$ std. liter $\min ^{-1}$ ) of $\operatorname{Ar}(99.999 \%$, Matheson) was added to convert He metastables and $\mathrm{He}_{2}{ }^{+}$into $\mathrm{Ar}^{+}$, after which time the plasma consists of $\mathrm{Ar}^{+}(\sim 95 \%) / e^{-}$, with the remainder of the positive charge being $\mathrm{He}^{+}$and minor impurities from trace air and water. All flows are metered using mass flow controllers (MKS). The gas pressure in the flow tube was varied to maintain a total number density of $3.2 \times 10^{16} \mathrm{~cm}^{-3}$ (1 Torr at $300 \mathrm{~K}$ ), except at temperatures above $600 \mathrm{~K}$ where the density was slightly reduced to as low as $2.1 \times 10^{16} \mathrm{~cm}^{-3}$ in order to produce a sufficiently high plasma density.

The temperature of each apparatus was maintained by resistive heating elements arranged in three individually controlled zones along the length of the flow tube, monitored by temperature sensors along the flow tube walls. The temperature readings were previously checked against the buffer gas temperature using a thermocouple movable along the axis of the flow tube. In order to maintain a constant temperature gradient along the reaction region, the upstream end of the flow tube must be heated to an elevated temperature, $10 \%-20 \%$ above the reaction temperature.

Halfway down the flow tube, the reactant gas $\left[\mathrm{F}_{2}(99 \%)\right.$ $0.5 \%$ in $\mathrm{He}(99.999 \%)$, Nova Gas] is added to the buffer through an inlet (glass in the FALP; quartz in the HT-FALP) with multiple hollow needles facing radially to distribute the $\mathrm{F}_{2}$ more evenly across the flow tube. In the FALP, the inlet line runs inside the flow tube from the downstream end for $\sim 50 \mathrm{~cm}$ in order to thermalize the reactant gas to the wall temperature. The construction of the HT-FALP constrains the inlet line to run from the upstream end of the flow tube, requiring the reactant gas to pass through the elevated temperature of the preheated zone. The $\mathrm{F}_{2}$ in He mixture flow was typically 5-10 std. $\mathrm{cm}^{3} \mathrm{~min}^{-1}$ to produce a nominal $\mathrm{F}_{2}$ concentration in the flow tube on the order of $10^{11} \mathrm{~cm}^{-3}$. Electrons attached to the $F_{2}$ downstream of the inlet, and the resulting decay of electron density as a function of distance along the flow tube axis, was measured using a cylindrical Langmuir probe $(7.6 \mathrm{~mm} \times 0.025 \mathrm{~mm}$ diameter FALP; $7.0 \mathrm{~mm} \times 0.076 \mathrm{~mm}$ HT-FALP) centered on the axis of the flow tube. In the FALP, the Langmuir probe was movable from $15 \mathrm{~cm}$ before to $35 \mathrm{~cm}$ after the reactant inlet; in the HT-FALP the probe was movable from 1 to $30 \mathrm{~cm}$ after the inlet. Diffusive electron loss was measured similarly, but with no $\mathrm{F}_{2}$ added. The plasma velocity was measured by pulsing the microwave discharge and noting the arrival time at the Langmuir probe as a function of distance along the flow tube; typical ion velocities were $100 \mathrm{~m} \mathrm{~s}^{-1}$.

Attachment rate constants were derived in the normal manner [21] from the observed decay of electron density $[e]_{t}$ both in the presence and absence of $\mathrm{F}_{2}$, according to

$$
[e]_{t}=[e]_{0} \frac{v_{a} e^{-v_{a} t}-v_{D} e^{-v_{D} t}}{v_{a}-v_{D}},
$$

where $[e]_{t}$ is the electron density at time $t$ (time zero defined by the addition of $\left.\mathrm{F}_{2}\right), v_{D}$ is the diffusion rate, $v_{a}$ is the attachment rate, related to the attachment rate constant $k_{a}$ by $v_{a}=k_{a}\left[\mathrm{~F}_{2}\right]$, with $\left[\mathrm{F}_{2}\right]$ being the reactant concentration. $[e]_{0}$ was typically $10^{9} \mathrm{~cm}^{-3}$, with Eq. (1) being valid where $\left[\mathrm{F}_{2}\right]_{0} \gg[e]_{0}$, such that $\left[F_{2}\right]$ is largely unchanged by the reaction; however, the rate constant may also be derived numerically in any case where
$\left[\mathrm{F}_{2}\right]_{0} \sim[e]_{0}$. A small portion of the buffer flow was sampled through an aperture in a truncated nose cone at the downstream end of the flow tube and the product ions were monitored using a quadrupole mass spectrometer with an electron multiplier.

Prior to measurements in both instruments, passivation of the inlet lines and flow tube with $\mathrm{F}_{2}$ was required. When $\mathrm{F}_{2}$ was initially added to either instrument only ions resulting from wall contaminants were observed; e.g., $\mathrm{CF}_{3} \mathrm{SO}_{3}{ }^{-}$in the FALP from experiments using triflic anhydride performed several months prior; presumably, the $\mathrm{F}_{2}$ displaced these gases from the inlet line surfaces. The $\mathrm{F}_{2}$ in He mixture was flowed through the inlet lines for several hours during the day, and the inlets and flow tube were baked at night with only He flowing. As this cleaning process was repeated, ions from contaminants decreased and $\mathrm{F}^{-}$signal was observed, with, after several days, $\mathrm{F}^{-}$dominating the mass spectra. Derivation of the attachment rate constant is dependent on knowing the concentration of $F_{2}$ delivered to the flow tube; any fraction of $\mathrm{F}_{2}$ lost to reaction in the inlet line will in proportion artificially decrease the observed rate constant. Clean mass spectra are necessary to ensure that the nominal $\left[\mathrm{F}_{2}\right]$ calculated from the gas flow rates is reaching the afterglow, but are not alone sufficient as side reactions could yield species that either do not attach or attach to also yield $\mathrm{F}^{-}$.

The actual $\mathrm{F}_{2}$ concentration could be determined by using the same apparatus to measure an ion-molecule rate constant and comparing to a known value. However, the literature on ion-molecule kinetics involving $\mathrm{F}_{2}$ is nearly nonexistent, with no positive ion chemistry reported [22] and just a single prior measurement of an anion reaction, that with $\mathrm{SF}_{6}{ }^{-}[23,24]$. Unfortunately, the $\mathrm{SF}_{6}{ }^{-}$reaction was studied in the aim of bracketing the $\mathrm{SF}_{6}$ electron affinity, meaning that the primary question of interest was simply whether or not reaction with $\mathrm{F}_{2}$ proceeded, with the rigor of determining the true $\mathrm{F}_{2}$ concentration and the resulting rate constant unclear. As such, it would be a poor anchor for the present experiment. Fortunately, we observe in the present experiments an $\mathrm{ArF}^{+}$ product peak, which we determine must arise from

$$
\mathrm{Ar}^{+}+\mathrm{F}_{2} \rightarrow \mathrm{ArF}^{+}+\mathrm{F},
$$

proceeding at or near the calculated collision rate [25]. The calculated reaction exothermicity of (2) is $0.45 \mathrm{eV}$ using the G3B3 model chemistry [26]. No other species possible from reaction with $F_{2}$ in the inlet lines is likely to have a weak enough $\mathrm{F}$ bond $(<\sim 2 \mathrm{eV})$ to similarly react with $\mathrm{Ar}^{+}$. Additionally, no other product cation species was observed to increase with increased $F_{2}$ flow rate over the temperature range where data are reported. Therefore, the ratio of the measured rate constant of (2) to the collisional rate constant sets a lower limit on $\left[\mathrm{F}_{2}\right]$ at an equal fraction of the nominal $\mathrm{F}_{2}$ concentration. The rate constant of (2) was measured in the standard manner, following the decay of the $\mathrm{Ar}^{+}$signal as a function of $\left[\mathrm{F}_{2}\right]$ under pseudo-first-order conditions. The reaction time was determined from the measured distance between the neutral inlet and the nose cone at the end of the flow tube (44 cm FALP; $53 \mathrm{~cm}$ HT-FALP) and the measured ion velocity.

Both the ion-molecule and $F_{2}$ electron attachment rate constants were measured from 300 to $600 \mathrm{~K}$ in $100 \mathrm{~K}$ increments in the FALP and from 600 to $900 \mathrm{~K}$ in the 


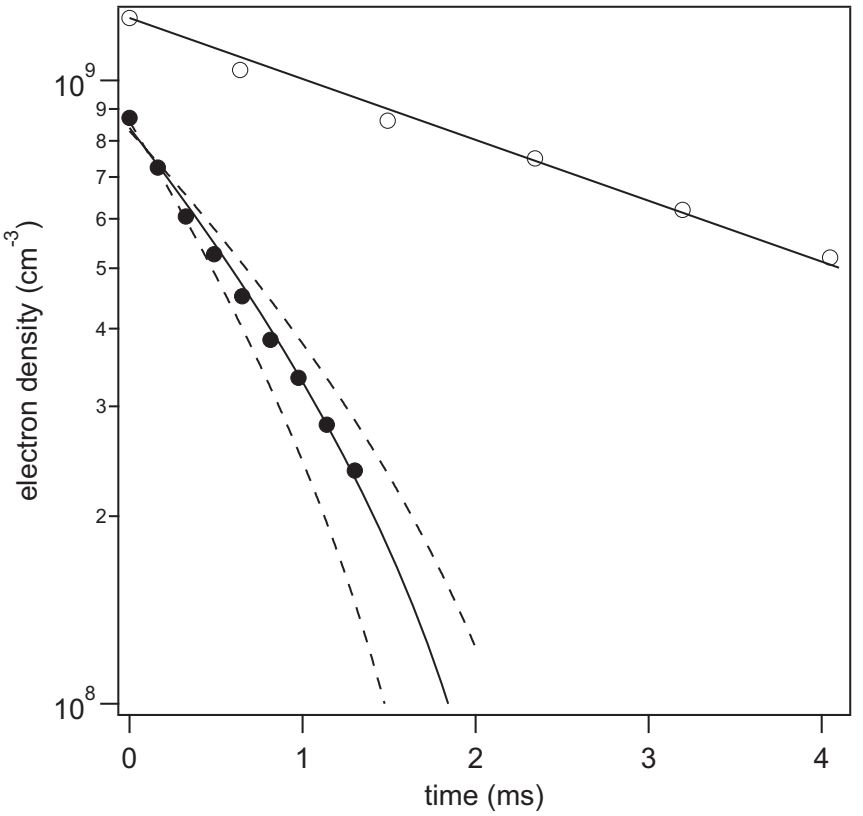

FIG. 1. Representative electron depletion as a function of reaction time due either solely to ambipolar diffusion (open circles; $\left[\mathrm{F}_{2}\right]=$ 0 ) or to both diffusion and attachment to $F_{2}$ (solid circles; $\left[F_{2}\right]=$ $10^{11} \mathrm{~cm}^{-3}$ ) at $300 \mathrm{~K}$. Solid lines are best modeled fits to the data; dashed lines are fits at the $25 \%$ uncertainty limits.

HT-FALP. At $800 \mathrm{~K}$ and especially at $900 \mathrm{~K}$, the measured electron attachment rate constants showed sharp drops relative to the lower temperature data, suggesting decomposition of $F_{2}$, likely through reactions on the walls of the inlet line. The ion-molecule measurements confirmed that the $\mathrm{F}_{2}$ was being destroyed in the inlet at those temperatures, with contaminant product cations rising with the $F_{2}$ flow rate. Continued passivation did not measurably improve the situation, and the highest temperature measurements were abandoned. Therefore, rate constants are reported from 300 to $700 \mathrm{~K}$.

\section{EXPERIMENTAL RESULTS}

Representative data from a single measurement of the $\mathrm{F}_{2}$ electron attachment rate constant appear in Fig. 1. The major source of systematic uncertainty is an accurate determination of the $\mathrm{F}_{2}$ concentration reaching the afterglow. At each temperature three to ten measurements were made over an extended period of time (between $1 \mathrm{~h}$ and $4 \mathrm{~d}$ ) to ensure that continued passivation with $\mathrm{F}_{2}$ showed no effect on the measured rate constant. Additionally at each temperature just before or after the attachment measurement, the rate constant of (2) was measured in order to set a lower limit on the $F_{2}$ concentration as described above. The values are shown in Fig. 2. In the FALP apparatus, the rate constant of (2) was $5.1 \times 10^{-10} \mathrm{~cm}^{3} \mathrm{~s}^{-1}$ at $300-500 \mathrm{~K}, 83 \%$ of the calculated collisional value [25] of $6.2 \times 10^{-10} \mathrm{~cm}^{3} \mathrm{~s}^{-1}$ (the collisional value is temperature independent owing to the zero dipole moment of $\mathrm{F}_{2}$ ). At $600 \mathrm{~K}$, the apparent rate constant of (2) fell to $4.1 \times 10^{-10} \mathrm{~cm}^{3} \mathrm{~s}^{-1}$. A sharp drop in the rate constant as observed is not realistic, and, while the uncertainty in the data does not rule out a small negative temperature

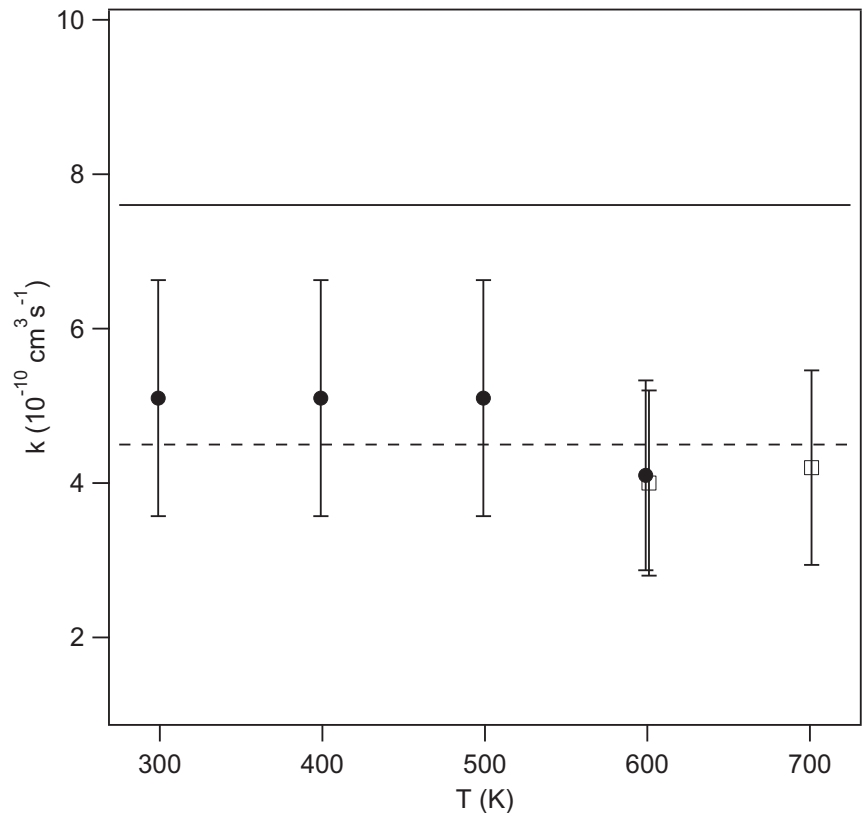

FIG. 2. Experimental rate constants for $\mathrm{Ar}^{+}+\mathrm{F}_{2} \rightarrow \mathrm{ArF}^{+}+\mathrm{F}$ at the indicated temperatures using the FALP (closed circles) and HT-FALP (open squares). Solid line indicates the calculated collision rate constant; dashed line indicates the recommended rate constant from 300 to $700 \mathrm{~K}$.

dependence, it appears more likely that the rate constant of (2) is near constant with temperature and the lowered $600 \mathrm{~K}$ value reflects loss of $F_{2}$ to reaction on the inlet line walls. As such, the measured $\mathrm{F}_{2}$ electron attachment rate constant at $600 \mathrm{~K}$ using the FALP has been corrected upwards by $20 \%$. The measured rate constants of (2) using the HT-FALP apparatus were $4.0 \times 10^{-10}$ and $4.2 \times 10^{-10} \mathrm{~cm}^{3} \mathrm{~s}^{-1}$ at 600 and $700 \mathrm{~K}$, respectively (no measurement was made at $500 \mathrm{~K}$ ). We attribute the observed deviation between the two apparatuses to end corrections in one or both measurements, with the apparent agreement at $600 \mathrm{~K}$ being only coincidental. At higher temperatures, a second product cation peak appeared at $m / Z=$ 85 , interpreted as being $\mathrm{H}_{2} \mathrm{FSO}_{2}{ }^{+}$, resulting from reaction of $\mathrm{F}_{2}$ on the inlet lines to form $\mathrm{HF}$ followed by reaction of $\mathrm{HF}$ with wall contaminants to form the precursor that underwent charge transfer from $\mathrm{Ar}^{+}$to yield $\mathrm{H}_{2} \mathrm{FSO}_{2}{ }^{+}$. We recommend a temperature-independent rate constant for (2) from 300 to $700 \mathrm{~K}$ of $4.5 \pm 1.5 \times 10^{-10} \mathrm{~cm}^{3} \mathrm{~s}^{-1}$. Although it appears that under most conditions the nominal $\mathrm{F}_{2}$ concentration was in fact reaching the afterglow, accounting for the possibility that (2) could proceed collisionally, our measured rate constants place a lower limit on the $\mathrm{F}_{2}$ concentration of $\sim 70 \%$ of the nominal value.

Figure 3 shows the measured rate constants for electron attachment to $F_{2}$ using the FALP and the HT-FALP and covering the temperature range of $300-700 \mathrm{~K}$. The data are tabulated in Table I. At all temperatures only DEA yielding $\mathrm{F}^{-}$was observed. The indicated uncertainties do not include the potential systematic uncertainty of overestimating $\left[\mathrm{F}_{2}\right]$, which would increase the rate constants by the amount of $\mathrm{F}_{2}$ lost. Because that would affect all values, the temperature dependence would barely change. Also shown in Fig. 3 are 


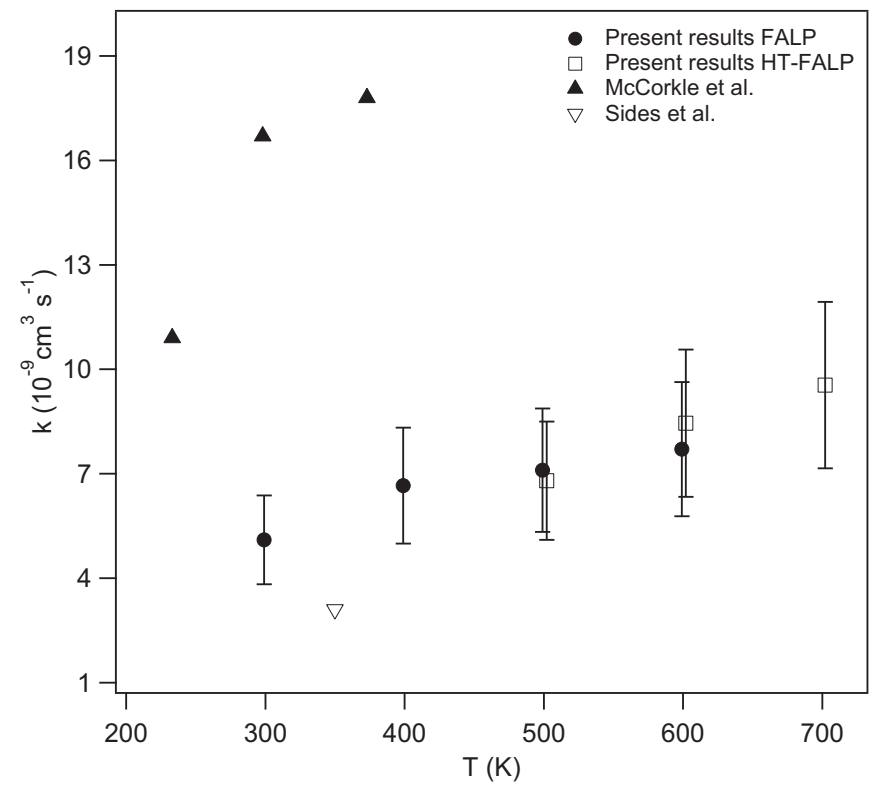

FIG. 3. Thermal rate constants for electron attachment to $F_{2}$. Solid circles and open squares are the present results using the FALP and HT-FALP, respectively. Solid triangles are from Ref. [16]; open triangle is from Ref. [17].

previously reported thermal results. McCorkle et al. measured energy dependences of the rate constants as a function of electron temperature as several temperatures [16]. These results at thermal temperatures are considerably larger than the present values. However, the swarm apparatus used to make those measurements did not have a mass spectrometer, and may well have been subject to the impurity issues we have discussed above. The Sides et al. datum was only quasithermal in that they estimated that the electron temperature was 350 or $600 \mathrm{~K}$ depending on the ion source [17]. While the results are in better agreement with the present values, the rate constants for several other reactions in that paper deviate significantly from now well-established literature values [7,27].

\section{IV. $R$-MATRIX CALCULATIONS}

The resonant $R$-matrix method employed in the present work is similar to that used in Refs. [4,6]. However, in contrast to those calculations, which were semiempirical, the $R$-matrix parameters here were chosen on the basis of previous DEA calculations for $F_{2}$. The potential energy curve for the

TABLE I. Measured rate constants for $\mathrm{F}_{2}+e \rightarrow \mathrm{F}+\mathrm{F}^{-}$; uncertainties are estimated at $\pm 25 \%$. This does not include a potential systematic error due to $F_{2}$ loss (see text) which would increase all rate constants by a similar amount.

\begin{tabular}{lcc}
\hline \hline & \multicolumn{3}{c}{$k\left(10^{-9} \mathrm{~cm}^{3} \mathrm{~s}^{-1}\right)$} \\
\hline$T(\mathrm{~K})$ & FALP & HT-FALP \\
300 & 5.1 & - \\
400 & 6.7 & - \\
500 & 7.1 & 6.7 \\
600 & 7.7 & 8.5 \\
700 & - & 9.6 \\
\hline \hline
\end{tabular}

neutral molecule was parametrized by the Morse potential using the known values of the vibrational quantum, $892 \mathrm{~cm}^{-1}$, the bond dissociation energy, $1.644 \mathrm{eV}$, and the equilibrium internuclear separation, $R_{e}=2.669$ a.u. For the anion curve, the Morse parameters were obtained by analysis of the ab initio data [12,28,29], which are all consistent with each other. The width calculations do not display such consistency. Moreover, there are qualitative differences in the results for the adiabatic width. Whereas calculations of Ingr et al. [28] demonstrate the validity of the Wigner threshold law $\Gamma \propto E^{l+\frac{1}{2}}$ for the case $l=$ 1 over a wide energy range of about $1 \mathrm{eV}$, Brems et al. [12] find its validity over only a very narrow interval of about $0.1 \mathrm{eV}$.

Note that the dynamical width necessary for nonlocal calculations was calculated in Refs. [11,12]. Both groups obtained very strong dependence of the dynamical width $\Gamma$ on the internuclear separation $R$ for a fixed electron energy $E$. This is surprising, because in the nonlocal theory $R$ plays the role of an adiabatic parameter, i.e., $\Gamma$ is assumed to be a slowly varying function of $R$. However, our model calculations have shown that results for the DEA cross sections are not substantially influenced by the rate of change of $\Gamma$ with $R$. This is confirmed by the calculations of Bardsley and Wadehra [10], who assumed that $\Gamma(R, E)$ is independent of $R$ at $R<3$ a.u. The most critical quantity is the value of $\Gamma$ at the stabilization point $R_{s}=2.61$ a.u., that is the point of crossing between the neutral and the anion potential energy curves. Note that as in the case of $\mathrm{Cl}_{2} R_{s}$ is smaller than $R_{e}$, although the difference is not as dramatic: $R_{S}$ lies well within the classically allowed region for the ground vibrational state of $\mathrm{F}_{2}$.

In the resonant $R$-matrix theory the resonance width $\Gamma$ is determined by the $R$-matrix surface amplitude $\gamma$. In order to reproduce the strong dependence of $\gamma$ on $R$ we parametrize it as

$$
\gamma(R)=\gamma_{0} e^{-\eta\left(R-R_{e}\right)},
$$

with the parameter $\eta$ between 1.0 and 1.5 a.u. Although we think that the choice $\eta>1.5$ a.u. is unrealistic, even the value $\eta$ $=1.5$ a.u. cannot quite reproduce the strong $\Gamma(R)$ dependence obtained in Refs. [11,12]. On the other hand, as mentioned above, this dependence does not influence results for cross sections noticeably. Much more critical is the choice of parameter $\gamma_{0}$. Unfortunately, Hazi et al. [11] do not provide a full description of $\Gamma(R, E)$, quoting only peak values of $\Gamma$ at several values of $R$ and stating that $\Gamma$ as a function of $E$ peaks typically at $E=1 \mathrm{eV}$. The function $\Gamma(R, E)$ given by Brems et al. [12] looks systematically lower than that of Hazi et al. by about a factor of 4.

Based on this information we have chosen three sets of parameters for the surface amplitude, Eq. (3). For the first set, called B1, $\gamma_{0}=0.09, \eta=1.5$; for the second, called $\mathrm{H} 1, \gamma_{0}=0.183, \eta=1.5$; and for the third, called $\mathrm{H} 2$, $\gamma_{0}=0.188, \eta=1.0$. B1 produces the cross sections shown in Fig. 4, consistent with those obtained by Brems et al. The second set produces the cross sections shown in Fig. 5. These are consistent with those obtained by Hazi et al. The cross sections obtained using parameter set $\mathrm{H} 2$ are very close to those obtained with parameter set $\mathrm{H} 1$, confirming the statement that cross sections are insensitive to the $R$ dependence of the width, and are not shown here. 


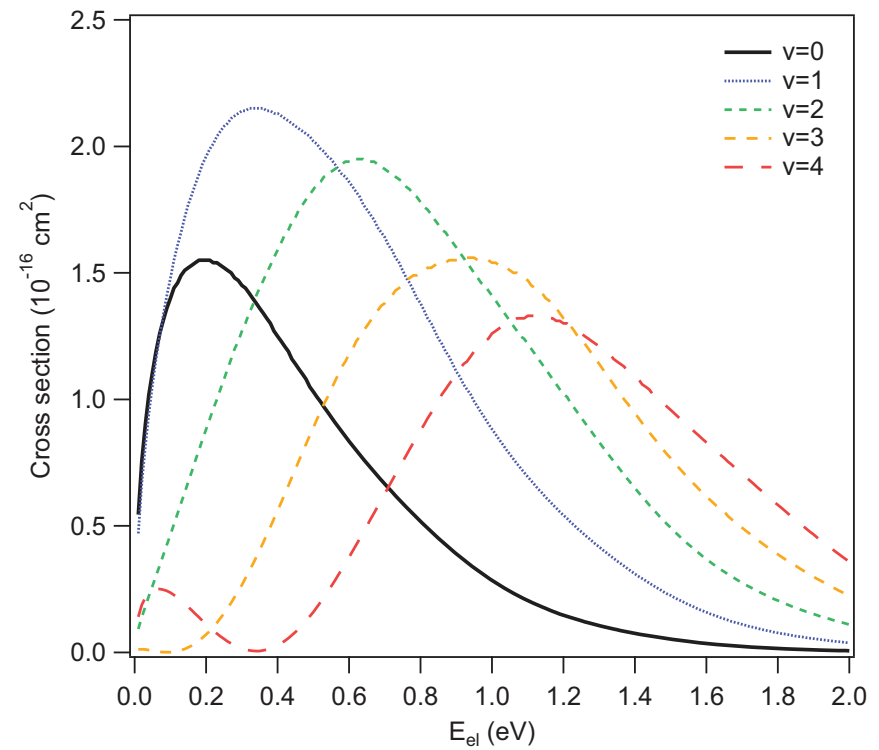

FIG. 4. (Color online) Cross sections for DEA to $F_{2}$ for the indicated vibrational states as a function of electron energy, corresponding to the model B1 with $\gamma_{0}=0.09$ and $\eta=1.5$.

In Fig. 6 we present the rate constants arising from the calculated cross sections as functions of gas temperature in comparison to the present experimental rate constants. The agreement between calculations using parameter sets $\mathrm{H} 1$ and $\mathrm{H} 2$ and experiment is exceptional, while the rate constants obtained from the parameters of set B1 are below the experiment. Parameter sets $\mathrm{H} 1$ and $\mathrm{H} 2$ produce substantially larger resonance widths and therefore substantially higher cross sections. Note that generally a larger width produces a larger electron capture amplitude, but a smaller survival factor. In case of the low-energy $s$-wave capture the second effect dominates; therefore larger width leads to smaller cross sections. However,

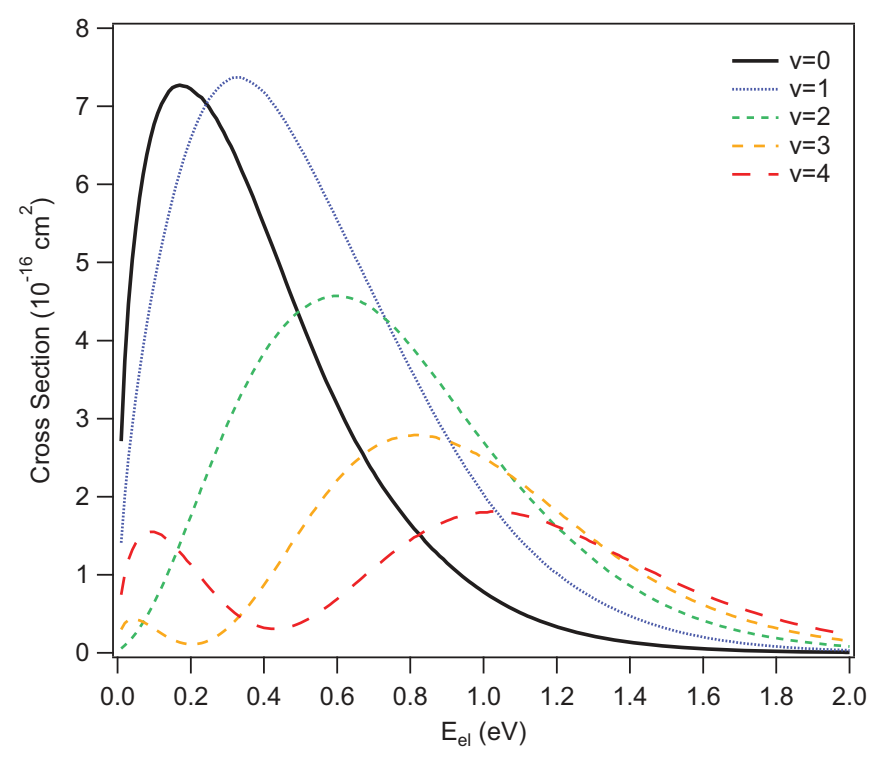

FIG. 5. (Color online) Cross sections for DEA to $F_{2}$ for the indicated vibrational states as a function of electron energy, corresponding to the model $\mathrm{H} 1$ with $\gamma_{0}=0.183$ and $\eta=1.5$.

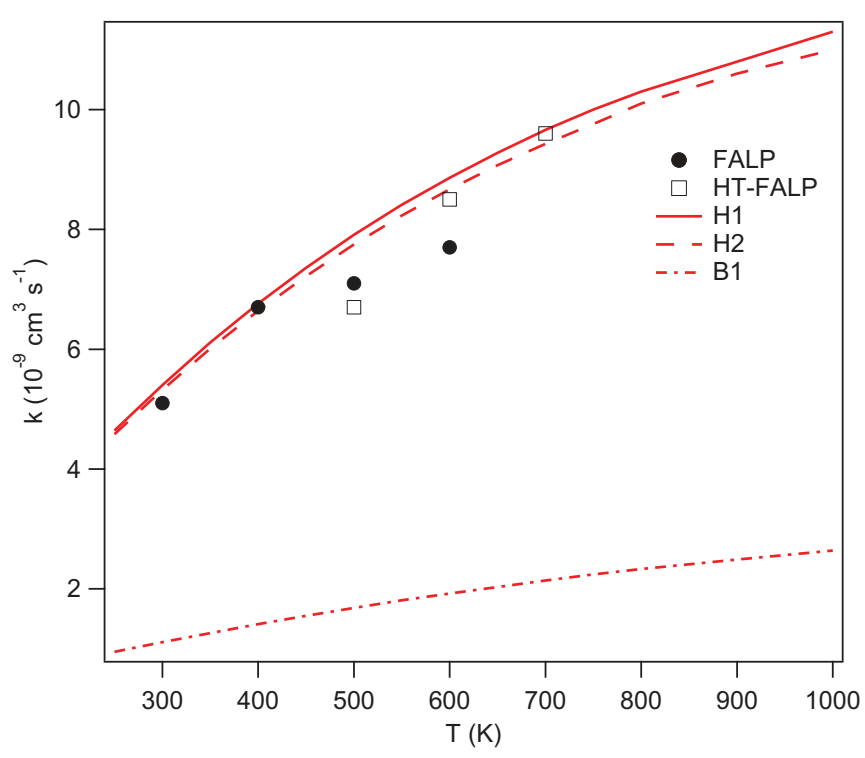

FIG. 6. (Color online) Calculated rate constants from the indicated models as functions of temperature, compared with the present experimental data.

in the present case of the $p$-wave capture the resonance width is relatively small, and the first effect becomes more important. It is noteworthy that the parameter sets $\mathrm{H} 1$ and $\mathrm{H} 2$ were fit to reproduce the width calculations of Ref. [11], not to reproduce the current experimental results, although the agreement is so good that it is likely, to some degree, fortuitous. The literature thermal rate constants reported by McCorkle et al. [16] are not reproducible through the $R$-matrix framework, even with attempts at freely varying the parameters. We conclude that the present measured rate constants allow us to decide which width calculations are more realistic.

\section{DISCUSSION}

The picture of electron attachment to $F_{2}$ at energies below $200 \mathrm{meV}$ electron energy remains muddled. Prior to the present work, results could be separated into two camps: those supporting $s$-wave threshold behavior and those supporting $p$ wave threshold behavior. The former consists of experiments by Chantry [1], Chutjian and Alajajian [2], and McCorkle et al. [16], which were all in quite reasonable agreement (see, for instance, Fig. 3 of Ref. [2], noting that the cross sections reported there by Chutjian and Alajajian were normalized to the $300 \mathrm{~K}$ result of McCorkle et al.). Recent local calculations by Honigmann et al. [13] exhibit very large cross section at low energies, although it is not clear if this high cross section demonstrates $s$-wave behavior as it is finite at zero energy. In any event, it is well known that threshold behavior inconsistent with the Wigner threshold law [3] is an artifact of the local theory. Support for $p$-wave threshold behavior consists of the recent high-resolution experiments by Braun et al. [8] and various calculations [10-12] all working from the assumption that attachment proceeds through the ${ }^{2} \Sigma_{u}^{+}$state. However, all attempts at reproducing the very-low-energy peak reported by Braun et al. through the $R$-matrix framework failed [9].

Discarding the thermal rate constant measurements of McCorkle et al. for the current values requires a 
renormalization of the cross sections reported by Chutjian and Alajajian, lowering the reported values by a factor of 3.6 and removing the pleasing agreement with the beam measurements of Chantry. The present results then perhaps leave a more clouded picture than they found, but do the service of correcting the literature in providing accurate thermal rate constants over a somewhat broad temperature range. That the results are in very good agreement with $R$-matrix calculations, and in fact the previous nonlocal calculations [11], provides support to that approach, which assumes $p$-wave threshold behavior.

The shape of the energy dependence of the cross section obtained by Braun et al. [8] cannot be reproduced by any calculations to date. Note that the measured cross section exhibits a minimum in the energy range between 130 and $140 \mathrm{meV}$, and then continues to grow as a function of $E$. It may be noted that the calculated cross sections of highly vibrationally excited $\mathrm{F}_{2}$ (Fig. 5) appears quite similar to the experimental results of Braun et al., but this must be coincidence as the $\mathrm{F}_{2}$ in the experiments was thermal. The question remains whether all theories developed so far are missing some physics allowing an explanation of the very-low-energy maximum and subsequent minimum in the attachment cross section observed by Braun et al.

\section{CONCLUSIONS}

Flowing afterglow apparatuses have been used to measure rate constants for thermal electron attachment to $F_{2}$ from 300 to $700 \mathrm{~K}$. The rate constant at $300 \mathrm{~K}$ is $5.0 \pm 1.3 \times 10^{-9} \mathrm{~cm}^{3}$ $\mathrm{s}^{-1}$ and is seen to increase with temperature rising to $9.6 \pm 2.4 \times 10^{-9} \mathrm{~cm}^{3} \mathrm{~s}^{-1}$ at $700 \mathrm{~K}$. The present rate constants are about a factor of 4 lower than the literature values of McCorkle et al. [16]. $R$-matrix calculations presented here are in excellent agreement with the current data. Notwithstanding this agreement, there remains a question about theoretical explanation of the behavior of high-resolution cross sections of Braun et al. for electron attachment to $F_{2}$. In the course of the experimental work, rate constants for the ion-molecule reaction $\mathrm{Ar}^{+}+\mathrm{F}_{2} \rightarrow \mathrm{ArF}^{+}+\mathrm{F}$ were measured $\left(4.5 \pm 1.5 \times 10^{-10} \mathrm{~cm}^{3} \mathrm{~s}^{-1}, 300\right.$ to $\left.700 \mathrm{~K}\right)$.

\section{ACKNOWLEDGMENTS}

We are grateful for the support of the Air Force Office of Scientific Research for this work under Project No. AFOSR-2303EP. T.M.M. is under contract (Contract No. FA8718-10-C-0002) to the Institute for Scientific Research of Boston College. I.I.F. was supported by the National Science Foundation, Grant No. PHY-0969381.
[1] P. J. Chantry, in Applied Atomic Collision Physics, Vol. 3: Gas Lasers, edited by H. S. W. Massey, E. W. McDaniel, and B. Bederson (Academic, New York, 1982), p. 35.

[2] A. Chutjian and S. H. Alajajian, Phys. Rev. A 35, 4512 (1987).

[3] E. P. Wigner, Phys. Rev. 73, 1002 (1948).

[4] I. I. Fabrikant, T. Leininger, and F. X. Gadea, J. Phys. B: At., Mol. Opt. Phys. 33, 4575 (2000).

[5] S. Barsotti, M. W. Ruf, and H. Hotop, Phys. Rev. Lett. 89, 083201 (2002).

[6] M. W. Ruf, S. Barsotti, M. Braun, H. Hotop, and I. I. Fabrikant, J. Phys. B: At., Mol. Opt. Phys. 37, 41 (2004).

[7] J. F. Friedman, T. M. Miller, L. C. Schaffer, A. A. Viggiano, and I. I. Fabrikant, Phys. Rev. A 79, 032707 (2009).

[8] M. Braun, M. W. Ruf, I. I. Fabrikant, and H. Hotop, Phys. Rev. Lett. 99, 253202 (2007).

[9] I. I. Fabrikant, J. Phys.: Conf. Ser. 204, 012004 (2010).

[10] J. N. Bardsley and J. M. Wadehra, J. Chem. Phys. 78, 7227 (1983).

[11] A. U. Hazi, A. E. Orel, and T. N. Rescigno, Phys. Rev. Lett. 46, 918 (1981)

[12] V. Brems, T. Beyer, B. M. Nestmann, H. D. Meyer, and L. S. Cederbaum, J. Chem. Phys. 117, 10635 (2002).

[13] M. Honigmann, R. J. Buenker, and H. P. Liebermann, J. Comput. Chem. 33, 355 (2012).

[14] W. Domcke, Phys. Rep. 208, 97 (1991).

[15] R. J. Hall, J. Chem. Phys. 68, 1803 (1978).

[16] D. L. McCorkle, L. G. Christophorou, A. A. Christodoulides, and L. Pichiarella, J. Chem. Phys. 85, 1966 (1986).
[17] G. D. Sides, T. O. Tiernan, and R. J. Hanrahan, J. Chem. Phys. 65, 1966 (1976).

[18] P. M. Hierl, J. F. Friedman, T. M. Miller, I. Dotan, M. MenendezBarreto, J. V. Seeley, J. S. Williamson, F. Dale, P. L. Mundis, R. A. Morris, J. F. Paulson, and A. A. Viggiano, Rev. Sci. Instrum. 67, 2142 (1996).

[19] T. M. Miller, A. E. S. Miller, J. F. Paulson, and X. F. Liu, J. Chem. Phys. 100, 8841 (1994).

[20] T. M. Miller, J. F. Friedman, J. S. Williamson, L. C. Schaffer, and A. A. Viggiano, Rev. Sci. Instrum. 80, 034104 (2009).

[21] D. Smith and P. Spanel, Adv. At. Mol. Opt. Phys. 32, 307 (1994).

[22] V. G. Anicich, JPL Report No. JPL 03-19 (2003).

[23] Y. Ikezoe, D. Matsuoka, M. Takebe, and A. A. Viggiano, Gas Phase Ion-Molecule Reaction Rate Constants Through 1986 (Maruzen, Tokyo, 1987).

[24] G. E. Streit, J. Chem. Phys. 77, 826 (1982).

[25] T. Su and W. J. Chesnavich, J. Chem. Phys. 76, 5183 (1982).

[26] Gaussian 09, Revision A.01, M. J. Frisch, G. W. Trucks, H. B. Schlegel, G. E. Scuseria, M. A. Robb, J. R. Cheeseman, G. Scalmani, V. Barone, B. Mennucci, G. A. Petersson et al. (Gaussian Inc., Wallingford, CT, 2009).

[27] J. Troe, T. M. Miller, and A. A. Viggiano, J. Chem. Phys. 127, 244304 (2007).

[28] M. Ingr, H. D. Meyer, and L. S. Cederbaum, J. Phys. B: At., Mol. Opt. Phys. 32, L547 (1999).

[29] T. N. Rescigno and C. F. Bender, J. Phys. B: At., Mol. Opt. Phys. 9, L329 (1976). 